\title{
Case Report \\ The Rare Malignancy of the Hepatobiliary System: Ampullary Carcinoid Tumor
}

\author{
Mustafa Ozsoy, ${ }^{1}$ Yucel Ozsoy, ${ }^{1}$ Aras Emre Canda, ${ }^{2}$ Olcay Ak Nalbant, ${ }^{3}$ and Fatih Haskaraca ${ }^{4}$ \\ ${ }^{1}$ Department of General Surgery, Manisa State Hospital, 45000 Manisa, Turkey \\ ${ }^{2}$ Department of General Surgery, Dokuz Eylul University School of Medicine, Izmir, Turkey \\ ${ }^{3}$ Department of Pathology, Manisa State Hospital, Manisa, Turkey \\ ${ }^{4}$ Department of General Surgery, Merkezefendi State Hospital, Manisa, Turkey \\ Correspondence should be addressed to Mustafa Ozsoy, dr.mustafaozsoy@gmail.com \\ Received 4 February 2011; Revised 9 April 2011; Accepted 12 April 2011 \\ Academic Editor: Robert A. Kozol
}

Copyright (๑) 2011 Mustafa Ozsoy et al. This is an open access article distributed under the Creative Commons Attribution License, which permits unrestricted use, distribution, and reproduction in any medium, provided the original work is properly cited.

Introduction. Carcinoid tumors are low-grade tumors originating from endoderm and mostly involving the gastrointestinal system. However; they may be seen in any site within the gastrointestinal system. Case Presentation. A 69-year-old female patient. The results of blood tests were observed to be consistent with obstructive jaundice. A mass appearance was not encountered on tomographic examination. Papilla that was tumor-like macroscopically was seen in the second part of the duodenum in diagnostic endoscopy. Pylorus - preserving pancreaticoduodenectomy surgical procedure was applied. On pathological examination of the mass, a tumoral mass was detected in ampulla vateri localization, $1.5 \times 1 \times 0.8 \mathrm{~cm}$ in size, which, in immunohistochemical staining, was evaluated as a neuroendocrine tumor. Also, Metastasis was observed. Conclusion. The rarest type of carcinoid tumor is ampullary located carcinoid tumor, and tumor size is not a reliable indicator for tumor aggressivity in ampullary carcinoid tumors.

\section{Introduction}

Carcinoid tumors are low-grade tumors originating from endoderm and mostly the involving gastrointestinal system. Although they may be seen at any site in the whole gastrointestinal system, the most commonly involved areas are appendix, distal small intestine, rectum, and stomach [1]. Herein, we described the rarest type of carcinoid tumor.

\section{Case Report}

A 69-year-old female patient was examined as jaundice was added to abdominal pain, accompanied by intermittent emesis and vomiting, lasting for 3 months. Results of blood tests were observed to be consistent with obstructive jaundice. A mass appearance was not encountered on tomographic examination obtained with prediagnosis of pancreatic cancer due to hydropic gallbladder and choledoc dilation on abdominal ultrasonography (Figure 1). Papilla that was tumor-like macroscopically was seen in the second part of the duodenum in diagnostic endoscopy (Figure 2). The patient, whose endoscopic biopsy result was evaluated as a well-differentiated endocrine tumor, was operated on. An ovoid mass $2 \times 2 \mathrm{~cm}$ in size was detected in ampulla vateri in intra-abdominal exploration. Pylorus - preserving pancreaticoduodenectomy surgical procedure was applied to the patient as the mass did not make vascular invasion but local invasion. The patient in whom a major surgical complication was not observed in postoperative followup was discharged in the postoperative second week. On pathologic examination of the mass, a tumoral mass was detected in ampulla vateri localization, $1.5 \times 1 \times 0.8 \mathrm{~cm}$ in size, and invaded submucosa and muscularis propria. The patient whose specimens were stained synaptophysin $(+)$, chromogranin-A $(+)$, and $\mathrm{Ki}-672 \%(+)$ in immunohistochemical staining was evaluated as a neuroendocrine tumor (Figure 3 ). Metastasis was observed in one out of 10 lymph nodes that were sorted out in the peripancreatic region of the patient whose surgical margins were healthy. No other foci were encountered in octreotide scintigraphy in the postoperative first month 


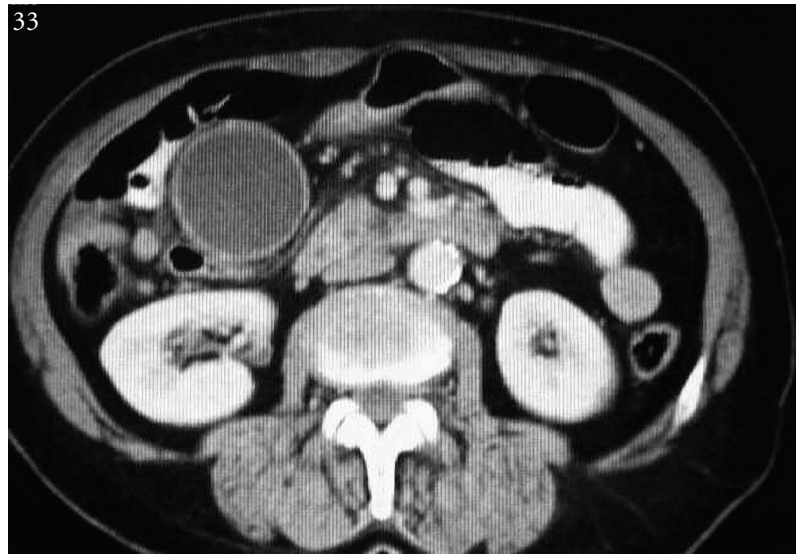

Figure 1: Hydropic gallbladder and choledoc dilation were seen on tomographic examination, Yet any mass appearance or lymph node was not encountered.

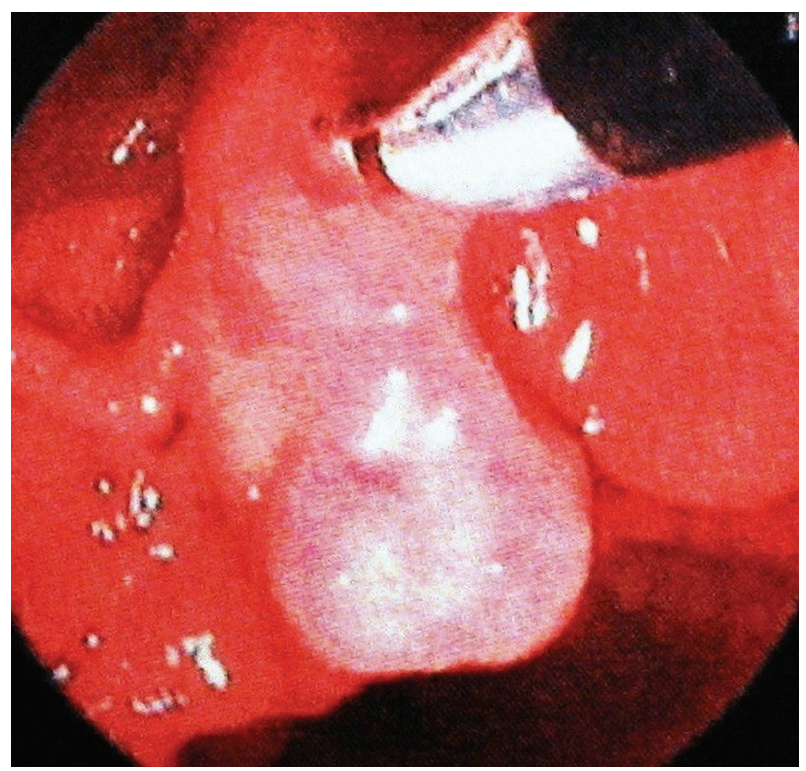

Figure 2: Papilla that was tumor-like macroscopically was seen in endoscopic retrograde cholangio pancreatography.

in the patient who was diagnosed as a well-differentiated endocrins tumor on histopathologic evaluation. Adjuvant chemotherapy and biologic treatment were not planned because of the absence of carcinoid syndrome findings and poor prognostic histopathologic properties.

\section{Discussion}

Ampullary located carcinoid tumor was first described by Oberndorfer in 1907 [2]. Although most of the reports are comprised of case reports, approximately 120 cases have been reported to date. It is rarer than even duodenal involvement defined as rare $(2 \%)$ with this number $(0.05 \%)[3,4]$. Its clinical presentation is composed of jaundice $(53,1 \%)$, abdominal pain $(24,6 \%)$, pancreatitis $(6,0 \%)$, and weight loss $(3,6 \%)[1-4]$. Abdominal tomography and endoscopic

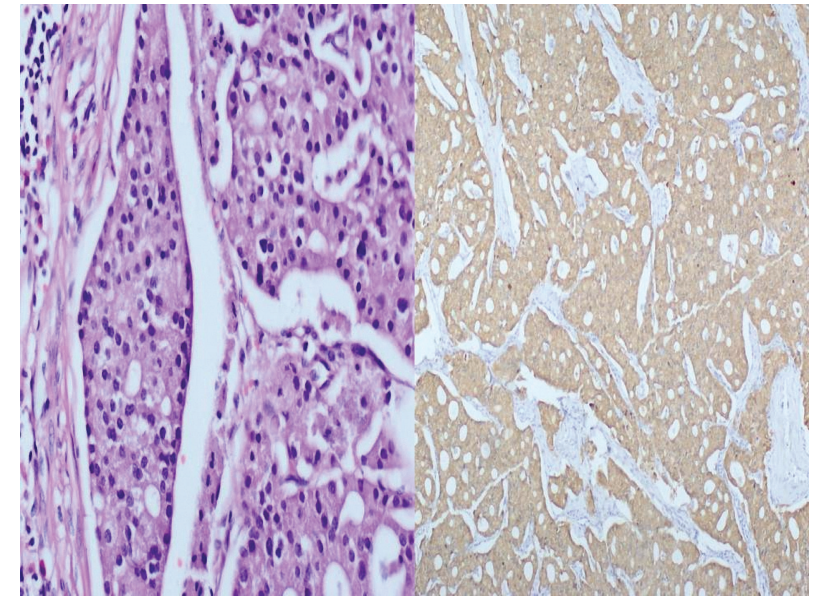

Figure 3: Specimens were stained synaptophysin (+), chromogranin-A (+), Ki-67 2\% (+) in immunohistochemical staining.

imaging methods are also valuable besides clinical findings for making a diagnosis. Of endoscopic imaging methods, especially endoscopic retrograde cholangio pancreatography, endoscopic biopsy and endosonographic ultrasonography may be used for the detection of invasion depth [5]. In 25\% of ampullary carcinoid tumors, they are seen to be related with von Recklinghausen syndrome and MEN syndromes. Additionally, most of the ampullary tumors have been seen to be positive in terms of somatostatin in pathologic examination. Thus octreotide scintigraphy is used with the aim of screening [6]. For carcinoid tumors with unknown malignity potential, a novel classification system was developed by the World Health Organization which classes tumors as benign, potentially malignant and malignant. In this classification, tumor proliferative index, invasion depth, and anatomic location were evaluated $[7,8]$. Ampullary carcinoid tumors still have obscurities about the issues like prognostic features, invasion depths, and lymph node metastasis because of its prevalence [9]. These obscurities also reflect on surgical treatment strategy of ampullary carcinoid tumors. Makhlouf et al. stated that metastatic disease was not in question for duodenal carcinoid tumors smaller than $20 \mathrm{~mm}$, whereas the same may not be said for ampullary carcinoid tumors. In literature, the presence of metastatic disease was seen in 40 $50 \%$ of ampullary carcinoid cases smaller than $20 \mathrm{~mm}$. Thus it is advocated that the most appropriate surgical treatment option should be the Whipple procedure $[9,10]$.

In our case, metastasis of lymph node was found although the tumor size was below $20 \mathrm{~mm}$, and all signs were not marked by the CT scan. Today, with the knowledge gained from oncologic surgical interventions, pancreaticoduodenectomy has become routinely practicable without any complications, which was inconceivable in the past. Therefore, we consider that, the most appropriate surgical treatment option for ampullary carcinoid tumors must be the Whipple procedure. Hwang et al. detected one-year survey as $90 \%$ and three-year survey as $64 \%$ in 10 patients who underwent pancreaticoduodenectomy with a mean tumor size of $2.1 \pm 1.3 \mathrm{~cm} \mathrm{[11]}$. 
The opponent group states that lymph node dissection does not have a therapeutic effect on ampullary carcinoid tumors and thus recommends local excision or endoscopic resection with lower morbidity and mortality $[12,13]$. However, as described by Clement et al., the possibility of residual tumoral formation is high with local resection, just as in the case that showed local recurrence 20 months after local excision [14]. These findings indicate that tumor size is not a reliable indicator for tumor aggressivity in ampullary carcinoid tumors. Adjuvant treatment options should be planned by taking prognostic factors of tumor and carcinoid syndrome findings into consideration. Adjuvant chemotherapy may be administered following resection in tumors that have poor prognostic factors. The most commonly used agents for chemotherapy are streptozocin, doxorubicin, dacarbazine, and 5-fluorouracil. Other treatment options are biologic therapy (interferon INF, somatostatin analogues SST) and chemoembolization $[13,14]$.

\section{Conclusion}

Gastrointestinal system-related deaths are placed highly among cancer-related deaths. Hepatobiliary system cancers are the most feared tumors among all gastrointestinal system tumors with a prevalence of 7-300/100.00 [15]. The only known potential therapeutic treatment option for these cancers is surgical RO resection. The presence of lymph node metastasis and tumor size is known as an independent prognostic factor for hepatobiliary cancers. However, tumor size is not a reliable indicator for tumor aggressivity in ampullary carcinoid tumors.

\section{Conflict of Interest}

The authors do not have any financial relationship with the organization that sponsored the research.

\section{References}

[1] M. Hartel, M. N. Wente, B. Sido, H. Friess, and M. W. Büchler, "Carcinoid of the ampulla of Vater," Journal of Gastroenterology and Hepatology, vol. 20, no. 5, pp. 676-681, 2005.

[2] S. Oberndorfer, "Karzinoide Tumoren des Dunndarms," Frank- furter Zeitschrift fur Pathologie, vol. 1, pp. 426-432, 1907.

[3] E. Hatzitheoklitos, M. W. Buchler, H. Friess et al., "Carcinoid of the ampulla of Vater: clinical characteristics and morphologic features," Cancer, vol. 73, no. 6, pp. 1580-1588, 1994.

[4] J. Waisberg, L. L. De Matos, D. R. Waisberg, H. V. B. Dos Santos, S. M. Fernezlian, and V. L. Capelozzi, "Carcinoid of the minor duodenal papilla associated with pancreas divisum: case report and review of the literature," Clinics, vol. 61, no. 4, pp. 365-368, 2006.

[5] F. Luka, I. Székely, C. Máj, I. Szántó, B. Hamvas, and A. Altorjay, "Carcinoid tumor of the papilla of Vater," Magyar Sebészet, vol. 58, no. 5, pp. 320-323, 2005.

[6] N. E. Koçer, F. Kayaselçuk, K. Çalişkan, and Ş. Ulusan, "Synchronous GIST with osteoclast-like giant cells and a well-differentiated neuroendocrine tumor in Ampula Vateri: coexistence of two extremely rare entities," Pathology Research and Practice, vol. 203, no. 9, pp. 667-670, 2007.

[7] E. K. G. Solcia and L. H. Sobin, Histological Typing of Endocrine Tumors, Springer, Heidelberg, Germany, 2000.

[8] G. Klöppel, P. U. Heitz, C. Capella, and E. Solcia, "Pathology and nomenclature of human gastrointestinal neuroendocrine (carcinoid) tumors and related lesions," World Journal of Surgery, vol. 20, no. 2, pp. 132-141, 1996.

[9] I. M. Modlin, M. Kidd, I. Latich, M. N. Zikusoka, and M. D. Shapiro, "Current status of gastrointestinal carcinoids," Gastroenterology, vol. 128, no. 6, pp. 1717-1751, 2005.

[10] H. R. Makhlouf, A. P. Burke, and L. H. Sobin, "Carcinoid tumors of the ampulla of vater: a comparison with duodenal carcinoid tumors," Cancer, vol. 85, no. 6, pp. 1241-1249, 1999.

[11] S. Hwang, S. G. Lee, Y. J. Lee et al., "Radical surgical resection for carcinoid tumors of the ampulla," Journal of Gastrointestinal Surgery, vol. 12, no. 4, pp. 713-717, 2008.

[12] D. K. Pyun, G. Moon, J. Han et al., "A carcinoid tumor of the ampulla of vater treated by endoscopic snare papillectomy," Korean Journal of Internal Medicine, vol. 19, no. 4, pp. 257260, 2004.

[13] J. L. Ricci, "Carcinoid of the ampulla of Vater: local resection or pancreaticoduodenectomy," Cancer, vol. 71, no. 3, pp. 686690, 1993.

[14] W. M. Clements, S. P. Martin, G. Stemmerman, and A. M. Lowy, "Ampullary carcinoid tumors: rationale for an aggressive surgical approach," Journal of Gastrointestinal Surgery, vol. 7, no. 6, pp. 773-776, 2003.

[15] C. F. Garland, F. C. Garland, and E. D. Gorham, "Etiology. Epidemiology end potantial prevention of the gastrointestinal cancer," in Surgery for Gastrointestinal Cancer, H. J. Wanebo, Ed., pp. 3-22, Lippincott-Raven, Philadelphia, Pa, USA, 1997. 


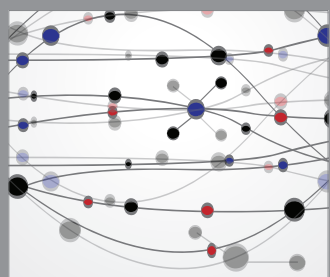

The Scientific World Journal
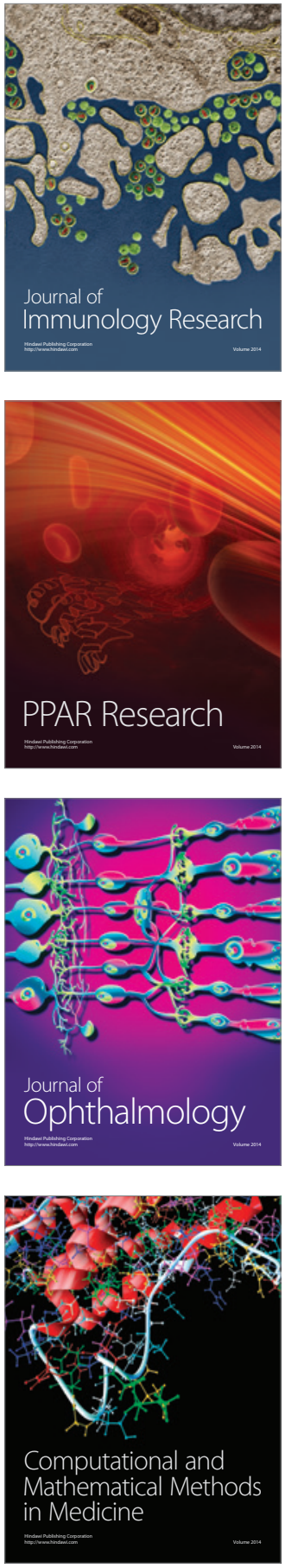

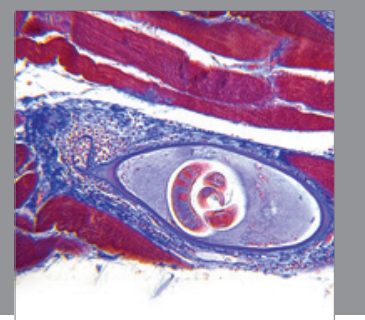

Gastroenterology

Research and Practice
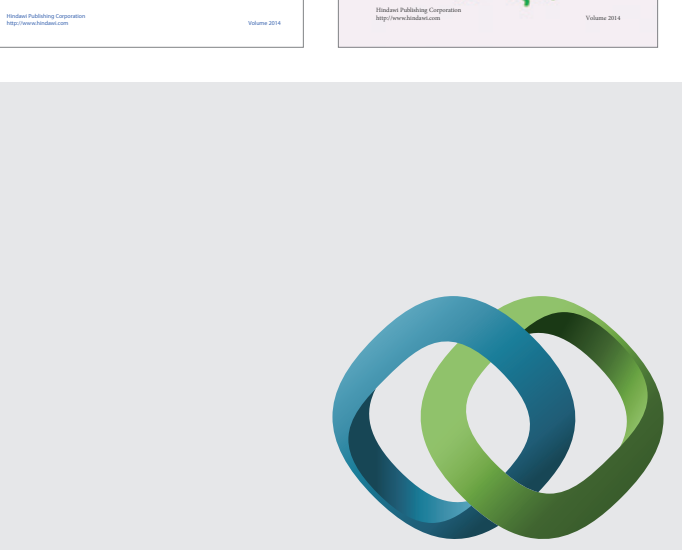

\section{Hindawi}

Submit your manuscripts at

http://www.hindawi.com
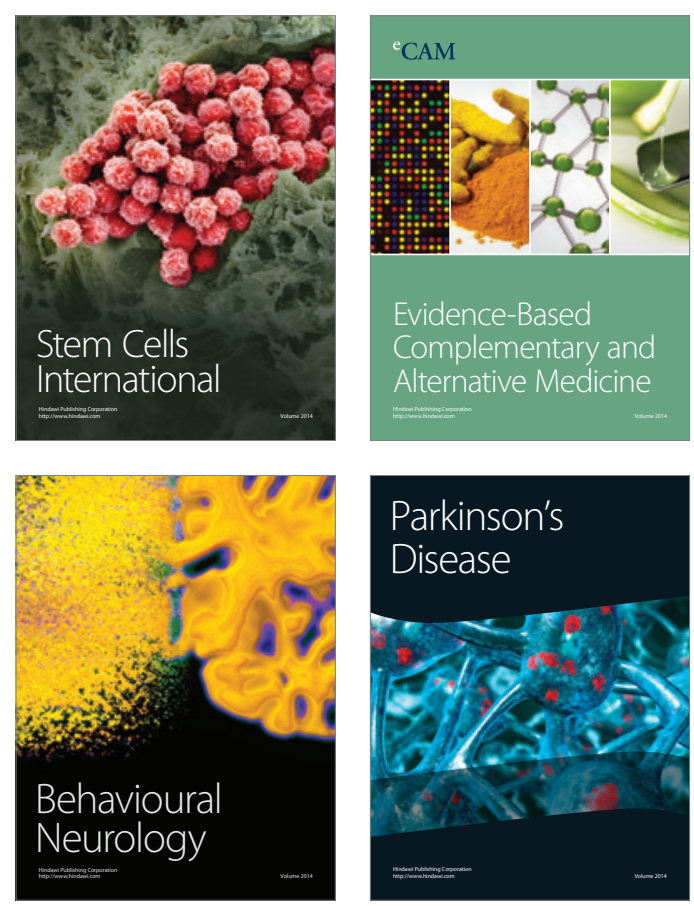

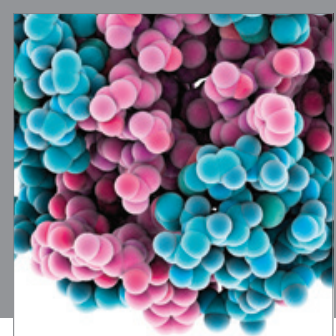

Journal of
Diabetes Research

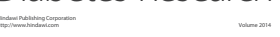

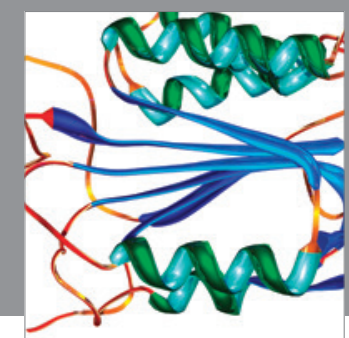

Disease Markers
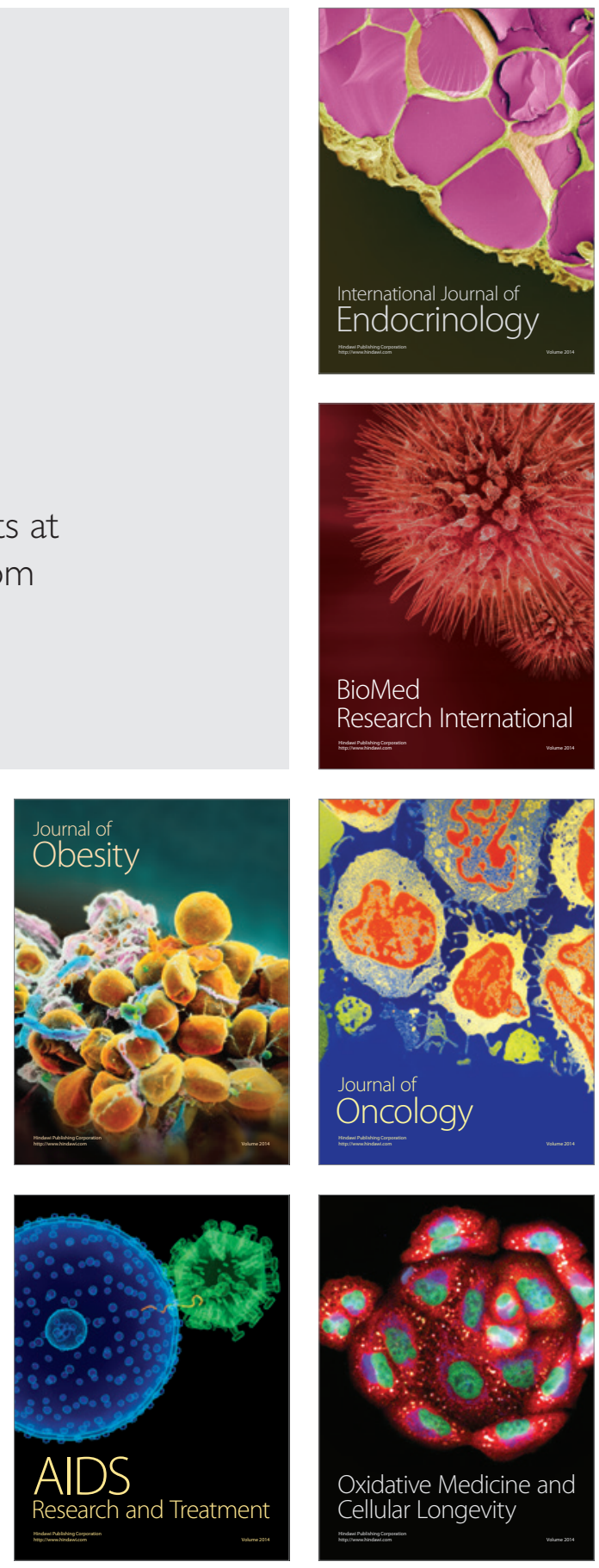